\title{
Formación de porosidad durante la soldadura láser de Nd:YAG de onda continua en aleaciones de aluminio para aplicaciones automotrices ${ }^{(\cdot)}$
}

\author{
Mario Pástor*, Hailiang Zhao** y Tarasankar DebRoy**
}

\begin{abstract}
Resumen La formación de poros es un aspecto de gran preocupación durante la soldadura láser de aleaciones de aluminio. En este trabajo se ha investigado la influencia del desenfoque del haz del láser sobre la formación de poros durante la soldadura láser (Nd:YAG de onda continua) de las aleaciones de aluminio 5182 y 5754, las mismas que se usan en aplicaciones automotrices. Los resultados obtenidos indicaron que la causa dominante de formación de poros fue la inestabilidad de la cavidad de vapor o keyhole mientras que la influencia del hidrógeno fue prácticamente insignificante. El grado de desenfoque del láser tuvo gran influencia sobre la inestabilidad de la cavidad de vapor. Finalmente, en este artículo se propone el mecanismo de formación de poros a causa del colapso de la cavidad de vapor (keyhole).
\end{abstract}

Palabras clave Formación de porosidad. Soldadura láser Nd:YAG. Aleaciones de aluminio.

\section{Pore formation during C.W.Nd:YAG laser welding of aluminum alloys for automotive applications}

\begin{abstract}
Pore formation is an important concern in laser welding of automotive aluminum alloys. This paper investigates the influence of the laser beam defocusing on pore formation during continuous wave $\mathrm{Nd}$ :YAG laser welding of aluminum automotive alloys 5182 and 5754 . It was found that the instability of the keyhole during welding was a dominant cause of pore formation while hydrogen rejection played an insignificant role. The defocusing of the laser beam greatly affected the stability of the keyhole. Finally, the mechanism of the collapse of the keyhole and pore formation is proposed.
\end{abstract}

Keywords Pore formation. Nd:YAG laser welding. Aluminum alloys.

\section{INTRODUCCIÓN}

Las características y propiedades de las aleaciones de aluminio, tales como su alta resistencia específica y a la corrosión, están moviendo a la industria hacia aplicaciones que tradicionalmente se basaron en el uso de aceros. La industria automotriz está soportando en la actualidad grandes presiones para que, simultáneamente, mejore el rendimiento en el uso de combustibles y reduzca las emisiones de gases que contribuyen a incrementar el efecto de invernadero. Es bien sabido que las aleaciones de aluminio tienen una resistencia específica superior a la del acero, por lo tanto, el uso de estas aleaciones es una alternativa que contribuiría a reducir notablemente el peso de las carrocerías y a cumplir estos nuevos estándares ${ }^{[1]}$. Sin embargo, uno de los mayores problemas para la implementación del aluminio en la construcción de carrocerías de automóviles es su soldabilidad cuando se usan procesos tradicionales de soldadura. El uso de la soldadura por resistencia ha demostrado ser el mejor para la fabricación de carrocerías de acero. Sin embargo, esta técnica de soldadura ya no es tan económica, tiene muchas limitaciones de acceso y no produce soldaduras de adecuada confiabilidad en aleaciones de aluminio. Adicionalmente, las altas velocidades de enfriamiento contribuyen a producir zonas térmicamente afectadas muy estrechas $^{[2]}$ y menor cantidad de hidrógeno disuelto en el metal fundido ${ }^{[3-6]}$. El uso del láser como fuente de energía para soldadura permite conseguir muy

(•) Trabajo recibido el día 16 de diciembre de 1999 y aceptado en su forma final el 14 de marzo de 2000.

$\left({ }^{*}\right)$ Escuela Superior Politécnica de Chimborazo, Facultad de Mecánica. Riobamba (Ecuador).

$\left(^{* *}\right)$ The Pennsylvania State University. Materials Science and Engineering Department. State College. Pennsylvania, PA (EE.UU.).

108

(c) Consejo Superior de Investigaciones Científicas

Licencia Creative Commons 3.0 España (by-nc) 
altas velocidades, excelente versatilidad para el posicionamiento del haz y completa automatización del proceso.

Uno de los problemas más críticos para la implementación de la soldadura láser en aleaciones de aluminio es la formación de poros, la pérdida de elementos de aleación y, en algunas aleaciones, la fisuración en caliente. Un problema adicional encontrado en las aleaciones de aluminio es el bajo coeficiente de absorción de la energía del láser. El coeficiente de absorción de la radiación del láser es una función de la longitud de onda; así, un láser de menor longitud de onda tendrá un mayor coeficiente de absorción y viceversa ${ }^{[7,8]}$. Duley ${ }^{[9]}$ demostró que para láser de $\mathrm{Nd}: \mathrm{YAG}$, con una longitud de onda característica de $1,06 \mathrm{~mm}$, el coeficiente de absorción en aluminio es mayor que el del láser de $\mathrm{CO}_{2}$, con una longitud característica de 10,6 $\mathrm{mm}$. Adicionalmente, se ha observado que la absorción de energía aumenta drásticamente cuando el haz de láser, tras formar una cavidad de vapor incide dentro de ella produciendo múltiples reflexiones internas ${ }^{[10]}$.

El efecto nocivo de los poros sobre las propiedades mecánicas de la junta soldada ha sido ampliamente expuesto en la literatura ${ }^{[11-13]}$. Sin embargo, el mecanismo de formación de poros durante la soldadura láser de aleaciones de aluminio no ha sido claramente explicado y no está bien comprendido. La formación de poros durante la soldadura láser ha sido vinculada a la diferencia de solubilidad del hidrógeno entre el aluminio líquido y sólido ${ }^{[14-17]}$, a la inestabilidad de la cavidad de vapor $^{[14,18,21]}$ y al flujo turbulento que ocurre en el pozo de soldadura ${ }^{[22]}$. El hidrógeno, que contaminará una soldadura, puede provenir del metal base, del metal de aporte o de otros contaminantes ${ }^{[23]}$. Woods ${ }^{[17]}$ demostró que para que se produzca porosidad es imprescindible que el contenido de este elemento en la soldadura esté siempre por encima de cierto valor, que dependerá de la concentración de elementos de aleación en el aluminio. Este resultado indica que si se reduce el contenido de hidrógeno, el nivel de porosidad en la soldadura también deberá reducirse. Sin embargo, experimentos realizados por Marsico ${ }^{[24]}$, demostraron que a pesar de que todas la posibles fuentes de contaminación fueron minimizadas, las soldaduras autógenas realizadas en aleaciones de aluminio con un haz de láser mostraron, siempre, un alto nivel de porosidad. Esta observación indica que el imperfecto colapso de la cavidad de vapor y las condiciones de flujo turbulento serían las causas más im- portantes de porosidad en la soldadura láser de estas aleaciones.

El propósito de este trabajo es investigar la influencia de los parámetros de soldadura sobre la formación de poros e identificar las causas de su formación durante la soldadura láser Nd:YAG de onda continua en aleaciones de aluminio-magnesio 5182 y 5754 . Estas son aleaciones representativas de las destinadas a la construcción de carrocerías de automóviles. La densidad de potencia del láser es uno de los parámetros más críticos para la soldadura. Así, la influencia de la densidad de potencia se evaluó soldando a varias posiciones de desenfoque del haz de láser.

\subsection{Características de la soldadura láser en aleaciones de aluminio}

Debido a la capacidad para concentrar energía en una pequeña área, el haz de láser es mucho más efectivo que cualquier otra fuente convencional de energía usada en soldadura, con excepción del haz de electrones. Así mismo, a diferencia del uso de haz de electrones, el haz de láser no es afectado por el campo magnético producido en la pieza de trabajo $^{[25]}$. La elevada densidad de potencia del láser produce un pozo de soldadura y una zona térmicamente afectada muy pequeños, combinados con grandes velocidades de calentamiento y enfriamiento en las regiones cercanas al pozo de soldadura. Estas condiciones producen una mínima distorsión de las soldaduras. La soldadura láser cubre una amplia gama de técnicas capaces de producir soldaduras con espesores que van desde unos cuantos micrómetros hasta varias decenas de milíme$\operatorname{tros}^{[26]}$.

Los dos tipos de láser más utilizados en el procesamiento de materiales y para soldadura son el láser de estado sólido, comúnmente conocido como Nd:YAG, y el láser de dióxido de carbono $\left(\mathrm{CO}_{2}\right)$. El láser de $\mathrm{Nd}$ :YAG tiene una longitud de onda de1,06 $\mathrm{mm}$ y puede desarrollar una potencia en el rango de $50 \mathrm{~W}$ a $4 \mathrm{~kW}$ con una eficiencia de aproximadamente $3,5 \%$ cuando se trabaja con un haz de onda continua ${ }^{[27]}$. El láser de $\mathrm{CO}_{2}$, por su parte, generara radiación infrarroja con una longitud de onda de 10,6 mm. Este tipo de láser puede producir una potencia superior a los $25 \mathrm{~kW}$ con una eficiencia en el rango de 7 a $10 \%{ }^{[28]}$. El uso de fibra óptica, como elemento de transmisión del haz de láser es muy conveniente debido a que se obtiene una elevada eficiencia de transmisión y porque permite gran facilidad de manipulación y 
posicionamiento del haz de láser de baja longitud de onda durante la soldadura. Nishimi et al. ${ }^{[29]}$ han informado que para el haz de láser de $\mathrm{Nd}$ :YAG, una fibra óptica puede transmitir el haz con una eficiencia del 92-93\%.

La densidad de potencia del láser debe seleccionarse de tal forma que asegure las condiciones a las cuales el régimen de entrada de energía supere el régimen de propagación del calor por conducción a través del metal. De esta manera, la energía suministrada por el láser rápidamente calentará la pieza a una temperatura superior a la de fusión y producirá un pozo de soldadura necesario para formar la junta soldada. La mínima densidad de potencia a la cual se produce una vaporización intensa de la mayoría de los metales varía entre $10^{5}$ a $10^{7} \mathrm{~W} / \mathrm{cm}^{2}$, dependiendo del calor de vaporización y de la conductividad térmica de cada metal ${ }^{[26,30]}$. El nivel de densidad de potencia deberá ser suficiente para que el pozo de soldadura adquiera una temperatura estable y, simultáneamente, el régimen de vaporización no sea excesivamente elevado. Con la soldadura láser se pueden realizar uniones usando altas velocidades de soldadura y sin necesidad de preparación de chaflanes. Adicionalmente, las juntas soldadas muestran mínima contaminación, y como resultado, la soldadura láser produce juntas de muy elevada calidad para muchas aplicaciones.

Gracias al desarrollo del láser de Nd:YAG de alta potencia, el uso de esta fuente de energía está experimentando un rápido proceso de expansión en el ámbito de la soldadura, incluyendo aplicaciones in situ para construcciones de gran tamaño, tales como barcos y plantas industriales donde hasta hace muy poco la aplicación del láser era muy restringida. La soldadura láser no está limitada a una única configuración de la junta. En láminas metálicas se pueden hacer soldaduras a tope, a solape, de borde, y en T, entre otras ${ }^{[29]}$. Se está implementando la soldadura láser para la fabricación de carrocerías de automóviles mediante la técnica denominada tailor blanks ${ }^{[31,32]}$. Esta es una técnica que consiste en soldar dos o más láminas de metal de distintas características mecánicas, espesores y/o revestimientos para producir una estructura de carrocería autoportante. Otras aplicaciones de la soldadura láser incluyen la fabricación de cárteres, filtros de combustible, partes de transmisión, zapatas de freno, columnas de dirección, embragues, convertidores de par y ruedas ${ }^{[29,33-34]}$. Un uso bastante intensivo de la soldadura láser se produce en la fabricación de paneles ligeros de piso y mampos- tería para la construcción de submarinos. Estas estructuras son construidas con lámina delgada para formar un conjunto liviano de alta resistencia.

La soldadura láser, al contrario de la soldadura por haz de electrones, no requiere del uso de alto vacío; se pueden soldar elementos de cualquier dimensión; la transferencia del haz es bastante simple y permite el uso de sistemas automáticos durante todo el proceso. Debido a que las aleaciones de aluminio tienen elevada difusividad térmica y elevada reflectividad óptica, la soldadura láser de estas aleaciones es bastante difícil. Al mismo tiempo, el bajo punto de fusión y la elevada fluidez son a veces una causa de problemas, tales como exceso de penetración y perforación de las partes que están siendo soldadas.

\subsection{Vaporización de la soldadura}

La elevada densidad de potencia utilizada para la soldadura láser en aleaciones de aluminio puede también producir vaporización de elementos de aleación de bajo punto de fusión tales como magnesio y zinc. Debido a que el magnesio tiene bajo punto de ebullición, este elemento puede ser fácilmente vaporizado del pozo de soldadura durante la soldadura láser, lo cual provoca una reducción de las propiedades mecánicas de la soldadura. Los resultados de Moon et al. ${ }^{[34]}$ mostraron que el contenido de magnesio en el pozo de soldadura de una soldadura láser realizada en una aleación 5456 fue de $4 \%$, mientras que el contenido de este elemento en el metal base fue de $5 \%$. Este resultado indica que la pérdida por evaporación en el pozo de soldadura fue del $20 \%$. Recientemente, Pástor et al. ${ }^{[35]}$ realizaron estudios de vaporización de magnesio en la aleación 5182. Sus resultados mostraron que la vaporización de magnesio dependía del modo de soldadura. Cuando la soldadura se realizó formando la cavidad de vapor, la pérdida por evaporación fue del orden del $16 \%$. Mientras tanto, en modo de conducción, la pérdida de magnesio fue del orden del $25 \%$. Este resultado muestra que, a causa de que en el modo de conducción hay una alta relación área de evaporación/volumen del líquido, la vaporización en este modo de soldadura láser es más intensa.

El mecanismo de vaporización fue estudiado por Collur et al. ${ }^{[36]}$ quienes explican que el proceso de vaporización puede dividirse en tres etapas. La primera consiste en el transporte de elementos hacia la superficie del metal en el pozo de soldadura. Durante la soldadura láser se produce 
una enérgica circulación del metal dentro del pozo de soldadura. Cálculos matemáticos muestran que la superficie del metal puede ser renovada 200 veces durante el tiempo en el que el haz del láser recorre una distancia igual al ancho del pozo de soldadura durante la soldadura láser de hierro puro ${ }^{[36]}$. Como la velocidad del flujo del metal en la soldadura láser de aluminio se considera mayor que en el caso del hierro, se puede asumir que la renovación de la superficie en el aluminio es mucho más rápida. Esta rápida renovación de la superficie indica que dentro del pozo de soldadura existe una mezcla muy enérgica por lo que no se observaría variación de concentración dentro del mismo. Este comportamiento fue demostrado por Pástor et al. ${ }^{[35]}$ quienes midieron los perfiles de concentración a lo largo de la soldadura. Aunque se encontró una importante variación de composición entre el metal base y el metal fundido, no se encontró variación de composición dentro de la zona de fusión. Una vez que los elementos de aleación llegan a la superficie, la vaporización queda determinada por algunas variables. Estas incluyen la distribución de la temperatura en la superficie, la concentración local de elementos de aleación y otros factores, como el agitamiento de la superficie y la presencia de plasma en la vecindad de la soldadura. La intensidad de vaporización de la superficie controla la pérdida total de elementos de aleación en el pozo de soldadura. Una vez que los elementos han sido vaporizados, su transporte desde la superficie de la soldadura hasta la fase gaseosa no retarda el proceso de vaporización.

\subsection{Defectos comunes producidos en la solda- dura láser de aleaciones de aluminio}

Durante la soldadura por fusión del aluminio y sus aleaciones, los problemas más comunes son porosidad, defectos geométricos y fisuras. Las fisuras de solidificación han sido observadas durante la soldadura láser de aleaciones de aluminio $2 \mathrm{xxx}^{[37,38]}$, $5 \mathrm{xxx}^{[39,40]}$ y $6 \mathrm{xxx}^{[41]}$. Por otro lado, muy pocas veces se ha observado la formación de fisuras de licuación. Jennings et al. ${ }^{[40]}$ establecieron que la susceptibilidad a la fisuración en caliente de las fundiciones de las aleaciones de aluminio depende de la composición. Está bastante bien probado que las aleaciones de las series $2 \mathrm{xxx}$ y $6 \mathrm{xxx}$ que son aleaciones térmicamente tratables son mucho más propensas a la fisuración que las aleaciones de la serie $5 \mathrm{xxx}$, que no son endurecibles por tratamientos térmicos. El uso de materiales de aporte ade- cuados también ha probado que puede reducir la formación de fisuras durante la soldadura láser de las aleaciones de las series $2 \mathrm{xxx}$ y $6 \mathrm{xxx}$. Mientras tanto, las aleaciones de la serie $5 \mathrm{xxx}$ pueden ser soldadas autógenamente usando un láser de onda continua sin que aparezcan fisuras en caliente ${ }^{[42]}$. Investigaciones recientes ${ }^{[13,43]}$ han revelado que la susceptibilidad a la fisuración en caliente de las aleaciones de aluminio durante la soldadura láser es muy sensible a los parámetros de soldadura utilizados. Cieslak et al. ${ }^{[43]}$ observaron fisuración en caliente en aleaciónes de aluminio 6061, 5456 y 5086 soldadas con un haz de láser pulsado de $\mathrm{Nd}$ :YAG, pero no observaron este problema cuando utilizaron un haz de láser de onda contínua de Nd:YAG. Katayama et al. ${ }^{[45]}$ también mostraron que la aleación de aluminio 2029 era más susceptible a la fisuración en caliente cuando se soldaba con un haz de onda pulsada que cuando se soldaba con un haz de onda continua.

El mecanismo de formación de poros durante la soldadura láser de aleaciones de aluminio no está totalmente entendido. Hay muy pocas investigaciones que tratan sobre la influencia de los parámetros de soldadura sobre la formación de poros. La influencia del hidrógeno y la estabilidad de la cavidad de vapor aparecen como los factores más influyentes sobre la formación de poros. En el caso del hidrógeno, este elemento puede formar poros esféricos de un diámetro de hasta varias decenas de micrómetros. Varios investigadores ${ }^{[3,5,45]}$ han demostrado que la formación de poros sólo es posible si la concentración de hidrógeno en el metal está por encima de cierto valor, que es función de la velocidad de enfriamiento. A medida que la velocidad de enfriamiento aumenta, la concentración de hidrógeno requerida para producir porosidad también aumenta. Aunque la mayor parte de estos estudios se realizaron para el caso de la fundición, en donde las velocidades de enfriamiento (1-20 ${ }^{\circ} \mathrm{C} / \mathrm{s}$ ) son mucho más lentas que las de soldadura, es de esperar que esta tendencia se mantenga. En la soldadura láser, las velocidades de enfriamiento son varios órdenes de magnitud mayores que las de la fundición; así, la concentración necesaria de hidrógeno para producir porosidad en el metal soldado debe ser muy elevada.

La influencia de la estabilidad de la cavidad de vapor sobre la formación de poros no está muy clara. En un trabajo previo ${ }^{[35]}$, se observó que la estabilidad de la cavidad de vapor es muy sensible a los cambios de energía de soldadura, la cual depende de las fluctuaciones de la energía del láser. Para un 
conjunto dado de parámetros de soldadura, la estabilidad de la cavidad de vapor es una función del balance de fuerzas que actúan dentro de esta. Las principales fuerzas que actúan en el interior de la cavidad de vapor se deben a la presión de vapor y a la tensión superficial. La magnitud de estas fuerzas son una función de la composición y temperatura de la aleación. Así, los parámetros de soldadura que son capaces de producir una cavidad de vapor estable son, también, una función de la concentración de elementos de aleación en el pozo de soldadura. Por consiguiente, los parámetros de soldadura necesarios para obtener cavidades de vapor estables y soldaduras libres de poros serán diferentes para cada aleación. Más aún, la pérdida de elementos de aleación debida a la vaporización producirá importantes cambios de composición en distintas regiones del pozo de metal líquido. De este modo, dependiendo de la magnitud de vaporización, la cavidad de vapor puede perder estabilidad y colapsar, atrapando gases o vapores y dando origen a la formación de poros.

\section{PARTE EXPERIMENTAL}

Se realizaron soldaduras autógenas utilizando un equipo de láser de estado sólido de Nd:YAG sobre placas delgadas de aleaciones de aluminio 5182 y 5754 , de $1,0 \mathrm{~mm}$ y $1,45 \mathrm{~mm}$ de espesor, respectivamente. Estos espesores se seleccionaron debido a que se consideran de potencial aplicación en la construcción de tailor blanks para carrocerías de automóvil. La composición química de las dos aleaciones se muestra en la tabla I. La potencia nominal de salida del láser se calibró para producir $3 \mathrm{~kW}$. El haz de láser se transmitió desde la salida del oscilador hasta la lente de enfoque, a través de una fibra óptica de $600 \mu \mathrm{m}$ de diámetro. Debido a que la eficiencia de transmisión de la fibra óptica ha sido calculada en $92 \%{ }^{[28]}$, la potencia efectiva del haz sobre la placa a soldar se estima que fue de $2,76 \mathrm{~kW}$. Para el enfoque del haz sobre la placa se

Tabla I. Composición química de las aleaciones de aluminio usadas en esta investigación (\% en peso)

Table I. Chemical composition of the aluminum alloys used in this work (\% wt.)

\begin{tabular}{ccccc}
\hline Aleación & $\mathrm{Mg}$ & $\mathrm{Si}$ & $\mathrm{Mn}$ & $\mathrm{Zn}$ \\
\hline 5182 & 4,44 & 0,20 & 0 & \\
5754 & 2,82 & 0,0 & 0,45 & 0,02 \\
\hline
\end{tabular}

usó una lente f2 con una distancia focal de 77,7 $\mathrm{mm}$. Esta lente fue montada sobre un posicionador vertical de tornillo micrométrico y este, a su vez, sobre un dispositivo de movimiento lineal accionado por un motor de pasos. La velocidad de la soldadura se calibró controlando la velocidad de avance de este dispositivo de movimiento lineal. La cabeza de enfoque se orientó de tal forma que formaba un ángulo de $75^{\circ}$ con respecto a la placa para evitar que la reflexión del haz pueda producir daños a la lente, a la fibra óptica o a las dos. $\mathrm{Al}$ mismo tiempo, formando un conjunto con la cabeza de enfoque, se montó una boquilla de cobre de 8 $\mathrm{mm}$ a través de la cual se hizo pasar un flujo de $5,66 \mathrm{~m}^{3} / \mathrm{h}$ de helio con el objeto de producir una atmósfera apropiada para la soldadura y para eliminar vapores y plasma que pudieran formarse sobre el pozo de soldadura. Esta boquilla se instaló formando un ángulo de $30^{\circ}$ con respecto a la placa (Fig. 1).

El efecto del desenfoque del haz se evaluó posicionando el foco del haz de láser a varias distancias, en el rango comprendido entre $+2,0 \mathrm{~mm}$ hasta $-2,0 \mathrm{~mm}$, arriba y abajo, respectivamente, de la superficie de la lámina a soldar. En este trabajo, la designación de desenfoque positivo indica que el foco del haz se encuentra encima de la lámina, mientras que la designación de desenfoque negativo indica que el foco del haz se encuentra debajo de la superficie de referencia de la lámina. La velocidad de avance del haz se determinó mediante ensayos previos en los cuales, para la potencia

\section{DIRECCION DE MOVIMIENTO} DEL HAZ DE LASER

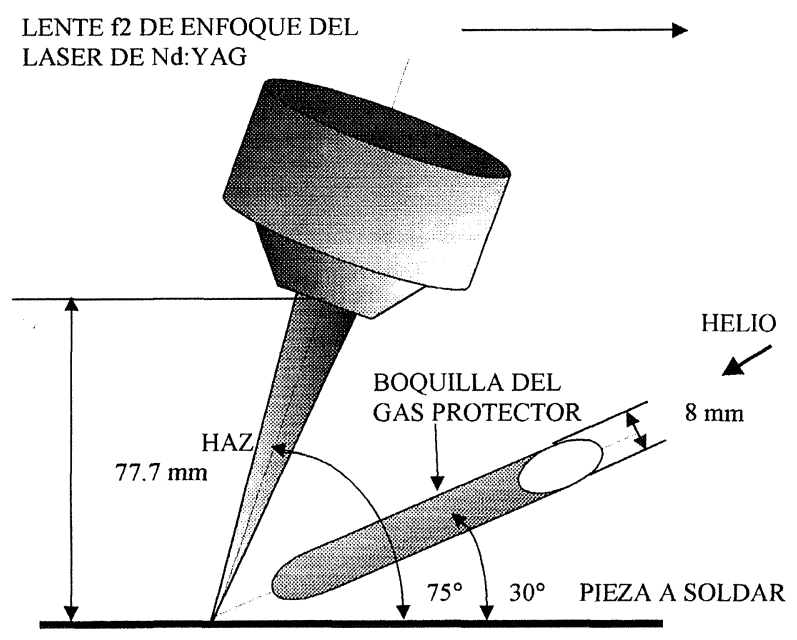

Figura 1. Esquema del montaje utilizado para la soldadura láser de Nd:YAG.

Figure 1. Nd:YAG welding arrangement. 
nominal de salida del haz de $3 \mathrm{~kW}$, se obtuvo penetración completa, sin exceso, de la soldadura en las placas de 1,0 y de $1,45 \mathrm{~mm}$, respectivamente. Las velocidades de $105,8 \mathrm{~mm} / \mathrm{s}$ para la soldadura de la aleación 5182 de $1,00 \mathrm{~mm}$ de espesor y de 63,5 $\mathrm{mm} / \mathrm{s}$ para la soldadura de la aleación 5754 de 1,45 $\mathrm{mm}$ de espesor fueron las apropiadas para este propósito. La geometría de la soldadura se determinó mediante el uso de microscopía óptica y análisis computarizado de imágenes. Los niveles de porosidad se midieron mediante el uso de microscopía electrónica de barrido para la caracterización de microporosidad y radiografía industrial, usando una fuente de rayos X para la caracterización de poros con dimensiones superiores a $200 \mu \mathrm{m}$.

\section{RESULTADOS Y DISCUSIÓN}

Debido a las técnicas usadas en esta investigación para detectar poros en la soldadura, los tipos de porosidad encontrada son microporos, con tamaños inferiores a $100 \mu \mathrm{m}$ pero también macroporos cuyas dimensiones son mayores de $100 \mu \mathrm{m}$. Los tipos de poros más comunes encontrados en las soldaduras se muestran en la figura 2.

El criterio de que el hidrógeno es la principal causa de porosidad durante la fundición y la soldadura de aleaciones de aluminio está ampliamente generalizado. La influencia de este elemento fue evaluada utilizando helio seco y húmedo como gas protector de la soldadura. El helio húmedo se consiguió haciendo pasar el gas a través de agua antes de ser usado sobre la soldadura. El helio húmedo se usó para evaluar la influencia de la concentración de humedad y por lo tanto de la concentración de

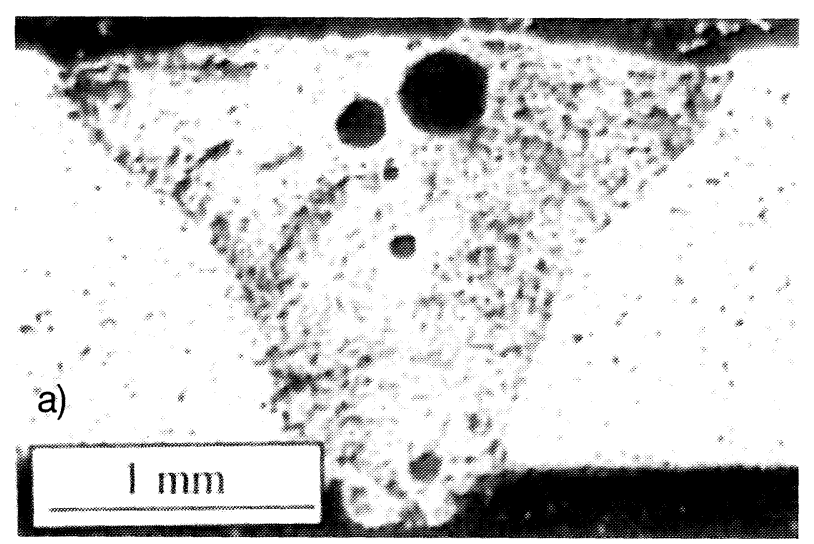

hidrógeno en el gas protector sobre la formación de porosidad en la soldadura. La presión parcial del vapor de agua en condiciones estándar es de 0,03 atm. Sin embargo, la presión parcial real del vapor de agua en el helio gas, después de pasar por el agua, fue de 0,008 atm. Este último valor se calculó considerando la pérdida de peso del agua en el dispositivo de burbujeo.

La figura 3 muestra los perfiles de las soldaduras realizados en la aleación 5754 a distintas posiciones de desenfoque en el rango de $-2,00 \mathrm{~mm}$ hasta $+2,00 \mathrm{~mm}$. En las figuras 3 y 4 se puede observar que al aumentar el desenfoque, la densidad de potencia del láser se reduce hasta alcanzar el valor mínimo necesario para que la soldadura se realice en modo de cavidad de vapor y, por consiguiente, al incrementar la extensión del desenfoque, el modo de soldadura por cavidad de vapor cambia súbitamente a modo de conducción.

Esta observación nos indica que existe una región de valores de desenfoque en la cual la cavidad de vapor puede ser altamente estable. Así, cuando el nivel de desenfoque origina la mínima densidad de potencia necesaria para abrir la cavidad de vapor, ésta es inherentemente inestable y puede ser causa de múltiples problemas en la soldadura.

La figura 3 también muestra que las secciones transversales de las soldaduras son bastante mayores para desenfoques negativos que para desenfoques positivos. La causa de este comportamiento sería que cuando el haz del láser se encuentra enfocado en el interior de la cavidad de vapor la absorción de energía del láser por el metal es mayor que cuando el haz se enfoca sobre la cavidad de vapor,

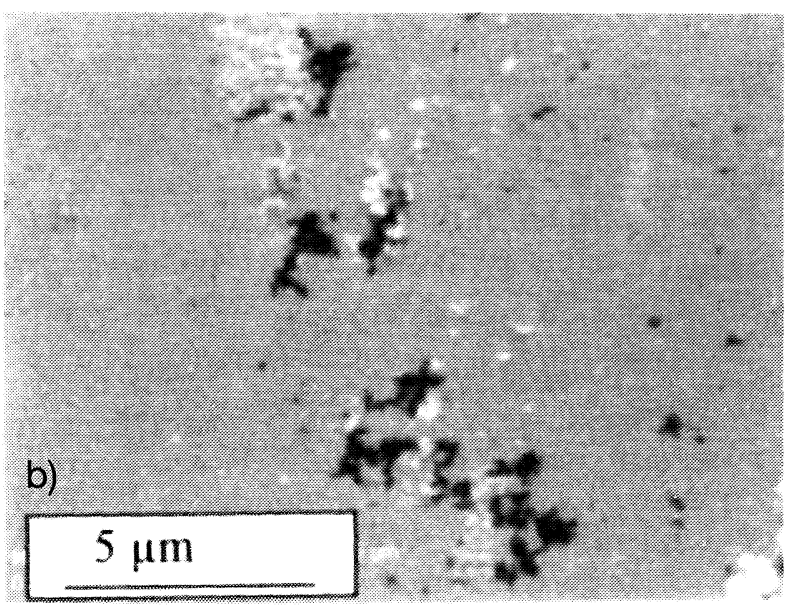

Figura 2. Secciones transversales de una soldadura en la aleación 5754 mostrando poros característicos producidos por la inestabilidad de la cavidad de vapor (a), y por contracción del metal durante la solidificación (b).

Figure 2. Cross sections of welds in alloy 5754 showing characteristic porosity produced by the instability of the keyhole (a) and by shrinking of the solidified metal (b). 


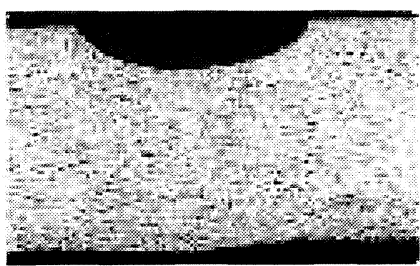

$-2,00 \mathrm{~mm}$

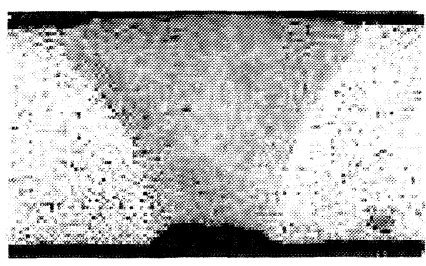

$-0,50 \mathrm{~mm}$

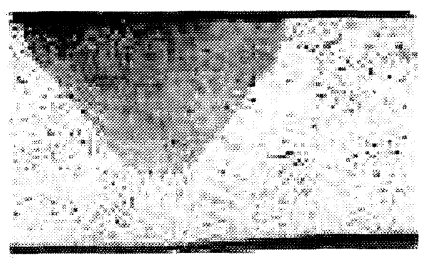

$+1,00 \mathrm{~mm}$

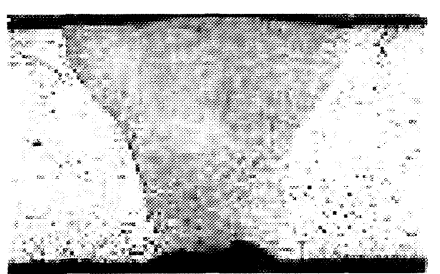

$-1,50 \mathrm{~mm}$

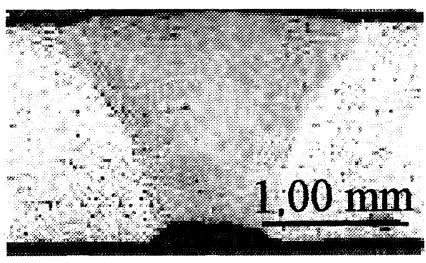

Enfocado

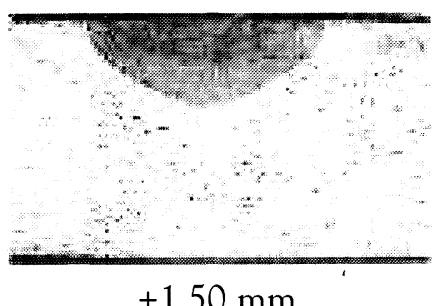

$+1,50 \mathrm{~mm}$

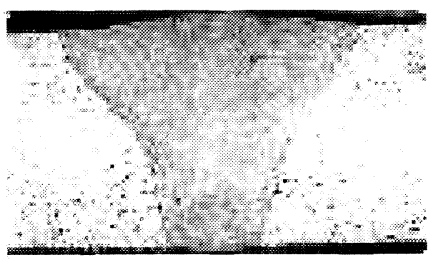

$-1,00 \mathrm{~mm}$

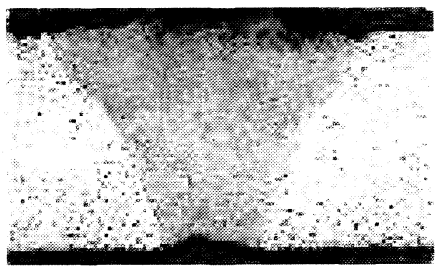

$+0,5 \mathrm{~mm}$

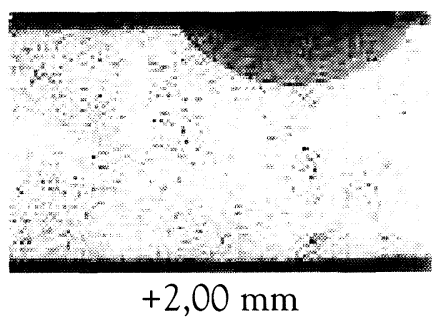

Figura 3. Secciones transversales de soldaduras realizadas en la aleación 5754 a varios valores de desenfoque en el rango comprendido entre $-2,00 \mathrm{~mm}$ hasta $+2,00 \mathrm{~mm}$. Velocidad del haz: 150 IPM $(63,5 \mathrm{~mm} / \mathrm{s})$. Flujo de gas protector: $200 \mathrm{CFH}\left(5,66 \mathrm{~m}^{3} / \mathrm{h}\right)$ de helio.

Figure 3. Cross section of 5754 alloy welds for the focus values in the range of $-2.0 \mathrm{~mm}$ to $+2.0 \mathrm{~mm}$. Welding speed: $150 \mathrm{IPM}(63.5 \mathrm{~mm} / \mathrm{s})$ and shielding gas flow rate: $200 \mathrm{CFH}\left(5.66 \mathrm{~m}^{3} / \mathrm{h}\right)$ of helium.

en donde una mayor fracción de la energía del haz es absorbida por el vapor para formar plasma. La figura 4 muestra que la profundidad de penetración de la soldadura cambia dependiendo del desenfoque utilizado.

Se puede observar en la figura 4 que los valores de penetración son reproducibles solamente cuando los niveles de desenfoque son próximos a cero, mientras que cuando el desenfoque supera ciertos valores, la penetración de la soldadura es aleatoria e irreproducible, lo cual es una indicación de inestabilidad de la cavidad de vapor. La figura 5 muestra el nivel de porosidad de la soldadura obtenido a varios valores de desenfoque en las aleaciones (a) 5182 y (b) 5754 cuando se usaron helio seco y helio húmedo como gas protector, respectivamente. El nivel de porosidad se presenta como porcentaje de volumen, el cual se calculó midiendo el volumen de macroporos presente en el metal depositado en la junta soldada.

En estas figuras, se observa que los menores niveles de porosidad corresponden a condiciones de soldadura en las que la cavidad de vapor era más estable, es decir, en el rango de $-1,0 \mathrm{~mm}$ hasta $+0,5 \mathrm{~mm}$. También se observan mínimos niveles de porosidad cuando, debido a un reducido nivel de densidad de potencia causado por altos valores de desenfoque, no se forma la cavidad de vapor en el pozo de soldadura, pero sí un pozo de metal líquido que da como resultado la soldadura en modo de conducción, y con ello, una geometría de forma de tazón. Esta forma del pozo de soldadura se observa cuando los valores de desenfoque fueron mayores de $-1,75 \mathrm{~mm} \mathrm{o}+1,5 \mathrm{~mm}$ para las dos aleaciones. Por el contrario, la mayor cantidad de poros fue encontrada en la región de transición, es decir, en la zona de desenfoque en la que la cavidad no era estable.

Comparando las curvas de niveles de porosidad obtenidas cuando se usó helio seco y helio húmedo, se observa que los niveles de porosidad detectados en el ensayo radiográfico en los dos casos son muy similares. Esta observación indica que la humedad inyectada a la soldadura no produce cambios significativos de porosidad.

Los ensayos de caracterización de poros que se realizaron usando microscopía electrónica de barrido con ampliaciones variables desde 100 aumentos hasta 10.000 sólo mostraron muy pocos poros de morfología irregular, la cual es característica del 


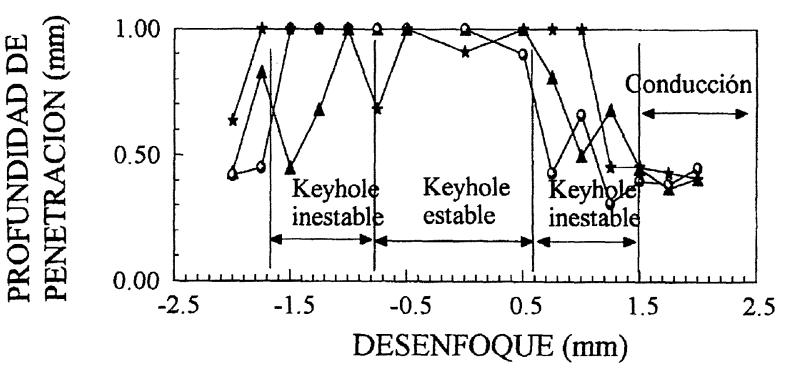

(a)

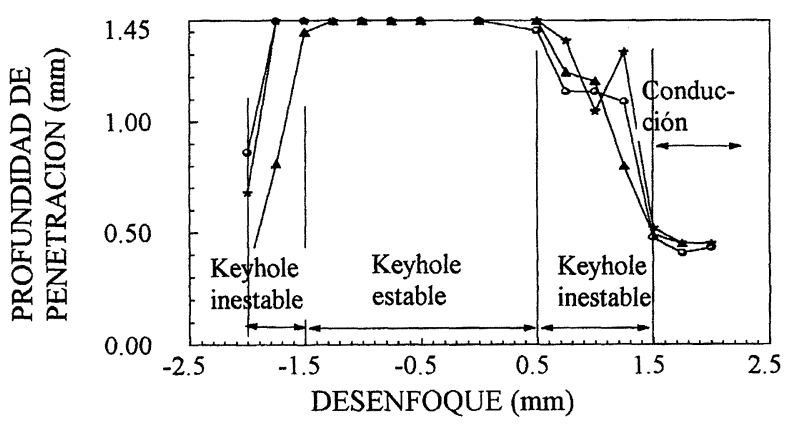

(b)

Figura 4. Variación de la profundidad de penetración de las soldaduras láser en las aleaciones 5182 (a) y 5754 (b) para varios valores de desenfoque. Potencia nominal: 3 $\mathrm{kW}$. Velocidad de soldadura $63,5 \mathrm{~mm} / \mathrm{s}$ y $105,8 \mathrm{~mm} / \mathrm{s}$, respectivamente. Flujo de gas protector: helio a $5,66 \mathrm{~m}^{3} / \mathrm{h}$. Las curvas representan tres grupos de experimentos.

Figure 4. Variation of the depth of penetration of the laser welds in alloys 5182 (a) y 5754 (b) for several defocus conditions. Nominal power: $3 \mathrm{~kW}$, welding speed, 63.5 $\mathrm{mm} / \mathrm{s}$. and $105.8 \mathrm{~mm} / \mathrm{s}$, respectively. Shielding gas flow rate: $5.66 \mathrm{~m}^{3} / \mathrm{h}$ of helium. Curves represent three sets of experiments.

proceso de solidificación. Estas observaciones indican que la inestabilidad de la cavidad de vapor es la principal causa de porosidad durante la soldadura láser $\mathrm{Nd}$ :YAG de aleaciones de aluminio-magnesio 5282 y 5754 . La segregación de hidrógeno, por su parte, jugó un papel marginal en la formación de poros en la soldadura de estas aleaciones. Esto se debe a que la velocidad de enfriamiento de la soldadura es demasiado rápida como para que el hidrógeno pueda ser absorbido en forma atómica, difundirse, formar hidrógeno molecular y, luego, crecer.

La estabilidad de la cavidad de vapor durante la soldadura láser depende en muy alto grado del balance de fuerzas que actúan en el interior de la cavidad de vapor ${ }^{[18]}$. Si la presión de vapor se reduce e iguala la presión debida a la tensión superficial, la cavidad pierde estabilidad y finalmente colapsa.

El mecanismo de formación de porosidad, debido a la inestabilidad de la cavidad puede expli-

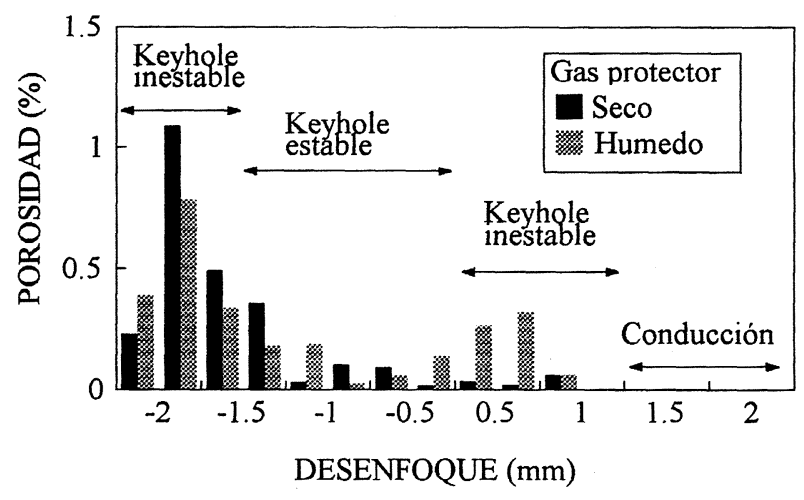

(a)

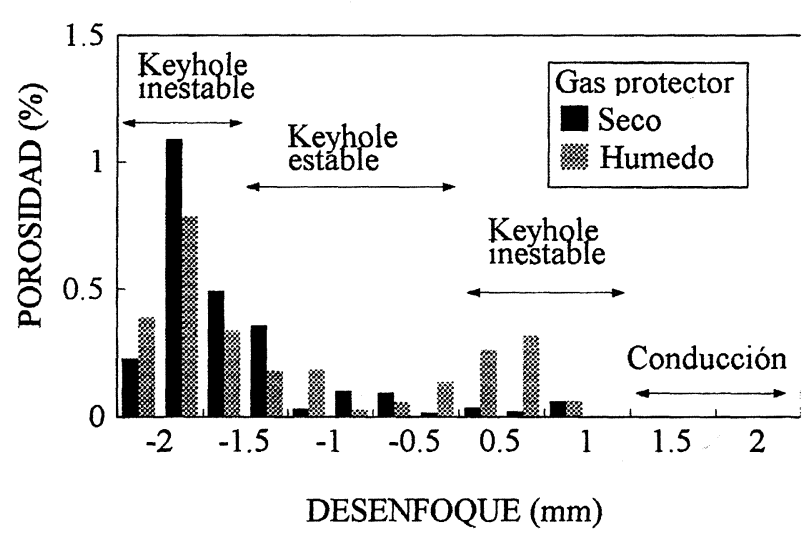

(b)

Figura 5. Porosidad producida a varios valores de desenfoque en las aleaciones (a) 5182 y (b) 5754 . Las curvas muestran el volumen total de porosidad obtenida: (i) helio seco usado como gas protector, y (ii) helio húmedo, es decir, burbujeado a través de agua. Potencia nominal: 3,0 $\mathrm{kW}$. Velocidad de soldadura: $250 \mathrm{IPM}(105,8 \mathrm{~mm} / \mathrm{s})$ para la aleación 5182 y $150 \operatorname{IPM}(63,5 \mathrm{~mm} / \mathrm{s})$ para la aleación 5754. Flujo de gas protector: $200 \mathrm{CFH}\left(5,66 \mathrm{~m}^{3} / \mathrm{h}\right)$.

Figure 5. Porosity produced at several defocus values in alloys (a) 5182 and (b) 5754. The curves depict the total volume of porosity obtained for: (i) dry helium used as a shielding gas and (ii) wet helium, i.e. bubbled through water. Nominal power: $3.0 \mathrm{~kW}$, welding speed: 250 IPM $(105.8 \mathrm{~mm} / \mathrm{s})$ for alloy 5182 and 150 IPM $(63.5 \mathrm{~mm} / \mathrm{s})$ for alloy 5754 , and shielding gas flow rate: $200 \mathrm{CFH}(5.66$ $\mathrm{m}^{3} / \mathrm{h}$ )

carse con la ayuda de la figura $6^{[21]}$. En esta figura se puede observar: (a) Cuando la presión debida a la tensión superficial supera la presión de vapor aparecen proyecciones en el interior de la cavidad. b) A medida que la presión de la tensión superficial crece por encima de la presión de vapor, el tamaño de la proyección aumenta. c) y d) La fuerza de gravedad hace que la proyección se mueva hacia la raíz de la cavidad. e) La proyección de metal líquido choca contra la cara opuesta y la cavidad atrapa gases y da origen a los poros. 


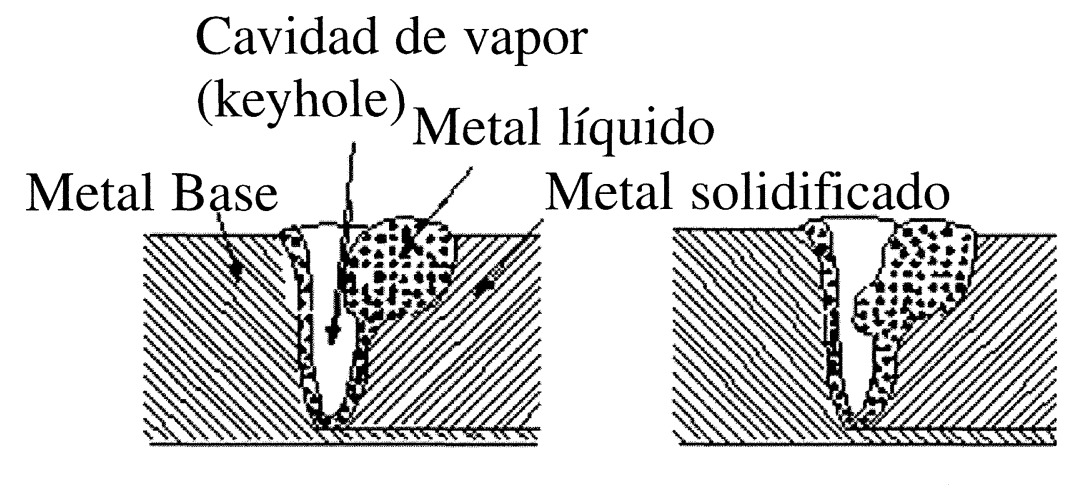

(a)

(b)

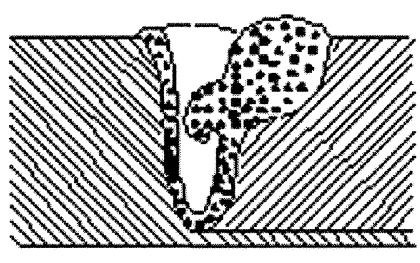

(c)

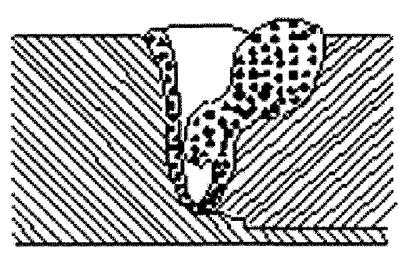

(d)

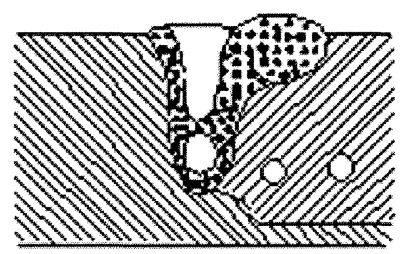

(e)

Figura 6. Esquema que explica el mecanismo de colapso de la cavidad de vapor y la formación de porosidad en la soldadura láser. (a) Cuando la presión debida a la tensión superficial supera la presión de vapor aparecen proyecciones en el interior de la cavidad. b) A medida que la presión de la tensión superficial crece por encima de la presión de vapor, el tamaño de la proyección aumenta. c) y d) La fuerza de gravedad hace que la proyección se mueva hacia la raíz de la cavidad. e) La proyección de metal líquido choca contra la cara opuesta y la cavidad atrapa gases y da origen a los poros.

Figure 6. Squematic drawing explaining the mechanism of the collapse of the keyhole and pore formation during laser welding. It is shown in (a) and (b) the formation of the liquid projection, in (c) and (d) the effect of the gravitational force on the liquid projection, and in (e) the collapse of the keyhole and pore formation.

\section{CONCLUSIONES}

Se ha estudiado la formación de poros durante la soldadura láser con cristal de Nd:YAG en dos aleaciones de aluminio para uso automotriz. Las principales conclusiones son las siguientes:

- Cuando los parámetros de soldadura se seleccionan de tal forma que se produce una condición de transición entre soldadura con formación de cavidad de vapor y soldadura en modo de conducción, aparecen poros con diámetros mayores de $0,2 \mathrm{~mm}$ aleatoriamente distribuidos a lo largo de la soldadura. Estos poros son resultado de la inestabilidad de la cavidad de vapor.

- La inestabilidad de la cavidad de vapor y la formación de poros pueden ser minimizadas ajustando apropiadamente el desenfoque del haz de láser.

- La influencia del hidrógeno sobre la formación de poros no fue un problema importante para la soldadura de estas aleaciones.
- Se propone un probable mecanismo de formación de poros debido a la inestabilidad de la cavidad de vapor.

\section{Agradecimientos}

Este estudio fue financiado por el U.S. Department of Energy, y FUNDACYT, Ecuador. Los autores agradecen al Dr. R.P. Martukanitz sus valiosos comentarios. Las soldaduras se realizaron gracias a la colaboración del Applied Research Laboratory, de la Pennsylvania State University. El material utilizado para los experimentos de esta investigación fue suministrado por Alcan International.

\section{REFERENCIAS}

[1] K.R. BROWN, M.S. VENIE y R.A. WOODS, JOM Julio (1995) 20-23.

[2] S.A. DAVID y J.M. VITEK, Int. Mater. Rev. 34 (1989) 213245. 
[3] M. Thomas y J.E. GruZleskl, Metall. Trans. B (1978) 139-141.

[4] H. VACCARI, Am. Mach. Febrero (1991) 50-52.

[5] E. Metcalfe, J. Inst. Met. 71 (1945) 618-619.

[6] E. Ransley y H. Neufeld, J. Inst. Met. 74 (1948) 599.

[7] M.A. Bramson, Infrared Radiation: A Handbook for Applications, Plenum Press, New York (EE.UU.), 1968, pp. 127.

[8] J.F. READY, Industrial Applications of Lasers. Academic Press, New York (EE.UU.), 1978, pp. 341.

[9] W.W. DulEY, Laser Processing and Analysis of Materials, Plenum Press, New York (EE.UU.), 1983, pp. 71.

[10] A. Huntington y T.W. Eagar, Weld. J. Res.Suppl. 62 (1983) 105s-107s.

[11] V. LAWRENCE Jr, WRC Bull. 181 (1973) 23-37.

[12] R.F. AsHTON Y R.P. WESLEY, Weld. J. 54 (1975) 95s-98s.

[13] M. КатоH, J. Light Met. Weld. Constr. 34 (1996) 42-48

[14] S. Katayama, J. Light Met. Weld. Constr. 34 (1996) 199-209.

[15] I. Masumoto y M. Kutsuna, IIW Doc. IV-566-91.

[16] M. KuTsunA, Weld. World 31 (1993) 126-135.

[17] R.A. WOODS, Proceedings of an international conference on the effects of hydrogen on materials properties. Selection and structural design. Ed. M. Bernstein y A.W. Thompson, ASM, Metals Park, OH, (EE.UU), 1974, pp. 713-725.

[18] D.A. Schauer y W.H. Giedt, Weld. J. 57 (1978) 189s195 s.

[19] I.R. Whitaker, D.G. McCartney y W.M. Steen, Proc. ICALEO. Orlando, Florida, FL (EE.UU.). Laser Institute of America/Society of Photo-Optical Instrumentation Engineers, 1992, pp. 565-573.

[20] R.P. Martukanitz y D.J. SMITH, Proceedings of the Sixth International Conference on Aluminum Weldments, Cleveland, OH, (EE.UU.), AWS, 1995, pp. 309-323.

[21] M. PÁstor, M.S. Tesis. The Pennsylvania State University, 1998.

[22] J.S. KIM, T. Watanabe e Y. Yoshida, J. Laser Appl. (7) (1995) 38-46.

[23] R.P. MaRTUKANITZ y P.R. MICHNUK, Aluminum, 58 (1982) 276-279.

[24] T. Marsico, M.S. Tesis. The Pennsylvania State University, 1988.

[25] A.G. GRIGORIANTS, Basics of laser Materials Processing, MIR Publishers/CRC Press, 1994, pp. 35.

[26] E.A. Metzbower, Weld. Res. Suppl. 72 (1993) $404 \mathrm{~s}-407 \mathrm{~s}$

[27] D.C WINBURN, What Every Engineer Should Know About Lasers, New York, NY (EE.UU.) Springer-PA, 1972, pp. 7-12.
[28] I.J. SPALDING, Proceedings of the 4th. International Conference of the Institution of Mechanical Engineers. Vol. 201. Núm. 80 (B3). Birmingham (Reino Unido), 1987., pp. 165-174.

[29] A. Nishimi, H. Kanazawa, Y. Taniu, K. Maeda e Y. WAKASA, Proceedings ICALEO. Laser Institute of America. Orlando, Florida, FL (EE.UU.), 1996, Sec. E, pp. 11-18

[30] ASM, Metals Handbook 9th edition, Vol. 6, Welding Brazing and Soldering, Laser Beam Welding, Metals Park, OH (EE.UU.), 1983, pp. 647-671.

[31] C.J. DAWES, Proceedings of LAMP'87, Osaka (Japón), 1987, pp. 323-328.

[32] P. Pecas, H. Gouveia, L. Quintino, M. Rasmussen y F.O. OLSEN, Rev. Metal. Madrid 34 (1998) 210-216.

[33] Y. Arata, H. Maruo, I. Miyamoto y S. Tacuchi, Plasma, Electron and Laser Beam Welding Technology, ASM Metals Park, Ohio, OH (EE.UU.), 1986, pp. 397.

[34] D.W. MoOn y E.A. Metzbower, Weld. J. 62 (1983) pp.53s-58s.

[35] M. Pástor, H. Zhao, R. Martukanitz y T. Debroy, Weld. J. (1999), 207s-216s.

[36] M.M. Collur, A. Paul y T. Debroy, Metall. Trans, 18B (1987), pp.733-740.

[37] S. Venkaty, C.E. Albright, S. Ramasaml y J.P. Hurley, Weld. J. 76 (1977) pp. 275s-282s.

[38] J.O. MILEWSKI, G.K. LEWIS y J.E. WITII, Weld. J. 1993, pp. $341 \mathrm{~s}-346 \mathrm{~s}$.

[39] A. Matsunawa, S. Katayama, H. Ikeda y K. Nishizawa: Proceedings ICALEO, 1992, Orlando, FL (EE.UU.) oct. 25-29. FL/Bellingham, WA. Laser Institute of America/Society of Photo-Optical Instrumentation Engineers, 1993, pp. 547-556.

[40] P.H. Jennings, A.R.E. Singer y W.I. PumphreY, J. Inst. Met. 74 (1948) 227-248.

[41] A. Blake y J. Mazumder, J. Eng. Ind. 107 (1985) 275280.

[42] R.P. Martukanitz, F.G. Armao, B. Altshuller y E.R. PICKERING, SAE Technical Paper 960168. Warrendale, PA (EE.UU.), Society of Automotive Engineers, 1996. Trabajo presentado al SAE International Congress and Exposition, Detroit, MI (EE.UU.), 26-29 feb. 1996.

[43] J. Cieslak y P.W. Fuerschbach, Metall. Trans. B, 19 (1988) 319-329.

[44] S. Katayama y C.D. Lundin, J. Light Met. Weld. Constr. 29 (1991) pp. 1-13.

[45] Q.T. Fang y D.A. Granger, TMS Annual Meeting of Light Metals, Las Vegas, Nevada (EE.UU.), 27 feb.-3 mar. 1989, TMS/AIME, Warrendale, PA (EE.UU.), pp. 927-935. 